Für die Zulassung zur Fachprüfung anerkannt werden ferner die in Zusammenarbeit mit der SGIM organisierten Fortbildungsveranstaltungen sowie die von der Fortbildungskommission SGIM für die obligatorische Fortbildung gemäss FBO-anerkannten Veranstaltungen mit einer Mindestdauer von 8 Stunden pro Tag/Einheit.

*Die Jahresversammlung wird administrativ von AKM Congress Service, Frau B. Meyer, Clarastrasse 57, 4005 Basel, Tel. 06168677 77, Fax 06168677 88, betreut. Anfragen über den wissenschaftlichen Teil aller Veranstaltungen und über organisatorische Belange betreffend die Fortbildungsveranstaltungen wollen Sie bitte an die jeweils oben erwähnten Verantwortlichen richten. Besten Dank.

Für generelle Fragen im Zusammenhang mit der Weiter- und Fortbildung in Innerer Medizin wenden Sie sich bitte an unsere Geschäftsstelle (diese kann aber nicht $\mathrm{zu}$ den einzelnen Veranstaltungen Stellung nehmen): SGIM, Postfach 158, 4011 Basel, Fax 061 22593 31, E-mail: info@sgim.ch.

\section{Informations au sujet de la formation post- graduée en vue de l'obtention d'un titre FMH en Médecine Interne}

\author{
Société Suisse de Médecine Interne
}

\section{Dispositions d'exécution relatives à l'art. 2.4 du Programme de formation postgraduée "Médecine Interne" du $1^{\text {er }}$ janvier 2000}

Justifier de la participation à une publication scientifique au moins:

- En plus de la thèse de doctorat requise selon l'art. 13 du Règlement sur la formation postgraduée de la FMH en vue de l'obtention d'un titre de spécialiste FMH (le Programme de formation postgraduée «Médecine Interne» est basé sur cet article du Règlement de formation postgraduée qui est prioritaire et ne comprend aucun règlement particulier sur la dissertation), la participation à une publication scientifique est exigée en tant qu'auteur principal ou coauteur. Sont acceptés, les travaux originaux traitant d'un thème de la médecine (sans restriction à des thèmes ou problèmes de la Médecine Interne), y compris la présentation de cas. Les compilations ne sont pas considérées comme travaux originaux.

- Le travail doit paraître dans une publication scientifique ou médicale.
- Les "abstracts" ne sont pas considérés comme publication scientifique, vu qu'il s'agit généralement de résumés de travaux scientifiques établis et soumis avant que ceux-ci n'aient été achevés.

- Si la thèse de doctorat a été publiée dans son entier, d'une manière élargie ou encore légèrement résumée dans une publication scientifique ou médicale, elle est considérée comme publication scientifique et répond donc aux exigences du § 1 de l'article 2.4.

Justifier de la participation à trois cours de formation continue au moins organisés par la Société Suisse de Médecine Interne (SSMI):

- Sont reconnus, les cours de formation continue organisés par la SSMI elle-même ou en collaboration avec celle-ci ainsi que les cours d'une durée de huit heures au moins, agréés par la Commission de formation continue de la SSMI comme répondant aux critères de formation continue obligatoire selon le Règlement de formation continue.

- Les principaux cours reconnus par la SSMI en tant que cours de formation continue sont publiés annuellement dans le Bulletin des médecins suisses et dans le Journal suisse de médecine.

\section{Cours de formation postgraduée et continue tenus en I'an 2000 et reconnus officiellement par la Société Suisse de Médecine Interne}

Les cours suivants sont reconnus officiellement pour l'an 2000 en tant que formation postgraduée valable pour l'admission à l'examen de spécialiste et pour l'octroi du titre FMH de Spécialiste en Médecine Interne:

Formation médicale continue Ligue pulmonaire, Zurich

13.-15.1.2000

Dr. Otto Brändli, tél. 05525661 11, fax 0552464720

Quadrimed, Crans-Montana

27.- 30.1.2000

Dr C. Vannay, tél. 02748562 28, fax 0274804055 
Assemblée générale de la SSMI*, Zurich

9. -11.3 .2000$

Prof. W. Vetter, tél. 0125524 09, fax 012554426

"Ärzteforum", Davos

12.- 17.3.2000

Prof. W. Reinhard,

tél. 08125662 05, fax 0812566381

"Lenker Woche"

3. - 8. 5. 2000

Dr. M. Essig, tél. 03372921 53, fax 0337292147

Cours officiel de formation continue de la SSMI, Berne

18. - 19.5. 2000

Prof. K. Neftel, tél. 03197071 11, fax 0319707763

Séminaire interdisciplinaire d'été, Davos

22.-28.7.2000

Prof. W. Siegenthaler. Davos Tourismus, tél. 08141521 21, fax 0814152101

Cours zurichois d'été de formation continue Lugano 2000

24.-26.8.2000

J. Ludin, Lungenliga Zürich, tél. 0126820 00, fax 012682020

$6^{\text {e }}$ Cours de formation continue de l'Engadine, Scuol-Vulpera-Tarasp

15.-17.9. 2000

Prof. C. Beglinger,

tél. 06126538 46, fax 0612653847
Cours général de formation continue de la Faculté de Médecine, Zurich

18.-22.9. 2000

Prof. W. Vetter, tél. 0125524 09, fax 012554426

Cours officiel der formation continue de la SSMI, Lucerne

10.-11.11.2000

PD Dr. V. Briner,

tél. 04120551 01, fax 0412055182

Sont également reconnus pour l'admission à l'examen de spécialiste, les cours de formation continue organisés en collaboration avec la SSMI ainsi que ceux d'une durée de huit heures au moins par jour ou par unité et agréés par la Commission de formation continue de la SSMI comme répondant aux critères de formation continue obligatoire selon le Règlement de formation continue.

*Madame B. Meyer de l'AKM Congress Service, Clarastrasse 57, 4005 Bâle, tél. 06168677 77, fax 061 6867788 est responsable administrativement de l'organisation de l'assemblée annuelle. Pour toutes questions relatives à l'aspect scientifique ainsi qu'à l'organisation des cours de formation continue, vous voudrez bien vous renseigner auprès de chacun des responsables mentionnés plus haut. Merci.

Pour des questions d'ordre général concernant la formation postgraduée et la formation continue en médecine interne, veuillez vous adresser à notre bureau de Bâle (qui ne peut cependant prendre position concernant un cours particulier): SSMI, Case postale 158, 4011 Bâle, fax 06122593 31, e-mail: info@sgim.ch. 